\title{
Non Progressive Disease
}

National Cancer Institute

\section{Source}

National Cancer Institute. Non Progressive Disease. NCI Thesaurus. Code C123599.

Following a previous assessment of progressive disease unconfirmed (PDu) of nontarget lesion(s), the subsequent required assessment does not confirm progressive disease. 